\title{
The relationship between abnormal Core binding factor- $\beta$ expression in human cartilage and osteoarthritis
}

\author{
Guangdi Li*, Mi Zhang, Yuan Huang, Jiafei Yang, Lianghong Dong, Hao Shi, Long Li, Riguang Liu and Jiangwei Li
}

\begin{abstract}
Background: This study aimed to investigate the effect of abnormal Core binding factor- $\beta$ expression on proliferation, differentiation and apoptosis of chondrocytes, and elucidate the relationship between Core binding factor- $\beta$ and osteoarthritis-related markers and degenerative joint disease.
\end{abstract}

Methods: Cartilage tissues, from healthy subjects and patients with osteoarthritis, were collected for histology and expression of Core binding factor- $\beta$, MMP-13, IL-1 $\beta$, COMP, and YKL-40. Human articular chondrocytes were cultured in vitro, and a viral vector was constructed to regulate cellular Core binding factor- $\beta$ expression. Cellular proliferation and apoptosis were observed, and osteoarthritis-related inflammatory factor expression and cartilage metabolite synthesis assayed.

Results: Human osteoarthritis lesions had disordered cartilage structure and cellular arrangement, and increased emptying of cartilage lacunae. Normal cell counts were significantly reduced, cartilage extracellular matrix was obviously damaged, and type II collagen expression was significantly decreased. Core binding factor- $\beta$ was highly expressed in the osteoarthritis cartilage $(p<0.001)$, and MMP-13, IL-1 $\beta$, COMP and YKL-40 expression were greater than found in normal cartilage $(p<0.001)$. Cellular proliferation in the Core binding factor- $\beta$ high-expression group was reduced and the total apoptosis rate was increased $(p<0.05)$, while the opposite was found in the Core binding factor- $\beta$ inhibition group $(p<0.01)$. Compared with normal chondrocytes, high Core binding factor- $\beta$ expression (Osteoarthritis and CBFB/pCDH groups) was associated with significantly increased MMP13, IL-1 $\beta$, COMP and YKL-40 protein expression $(p<0.01)$, while Core binding factor- $\beta$ inhibition (CBFB/pLKO.1 group) was associated with significantly decreased COMP, MMP13, IL-1 $\beta$ and YKL-40 expression in osteoarthritis cells $(p<0.001)$.

Conclusions: Abnormal Core binding factor- $\beta$ expression might play an upstream regulatory role in mediating abnormal chondrocyte apoptosis and the inflammatory response. On inhibiting Core binding factor- $\beta$ expression, a delay in cartilage degeneration was expected.

Trial registration: The study was registered for clinical trials in ChiCTR: ChiCTR1800017066 (Reg. Date-2018/7/10).

Keywords: Core binding factor- $\beta$, Osteoarthritis, MMP-13, IL-1 beta, COMP

\footnotetext{
* Correspondence: 3592487460@qq.com

Department of Orthopaedics, The Affiliated Hospital of Guizhou Medical

University, Guiyang, China
}

(c) The Author(s). 2021 Open Access This article is licensed under a Creative Commons Attribution 4.0 International License, which permits use, sharing, adaptation, distribution and reproduction in any medium or format, as long as you give appropriate credit to the original author(s) and the source, provide a link to the Creative Commons licence, and indicate if changes were made. The images or other third party material in this article are included in the article's Creative Commons licence, unless indicated otherwise in a credit line to the material. If material is not included in the article's Creative Commons licence and your intended use is not permitted by statutory regulation or exceeds the permitted use, you will need to obtain permission directly from the copyright holder. To view a copy of this licence, visit http://creativecommons.org/licenses/by/4.0/ The Creative Commons Public Domain Dedication waiver (http://creativecommons.org/publicdomain/zero/1.0/) applies to the data made available in this article, unless otherwise stated in a credit line to the data. 


\section{Background}

Osteoarthritis (OA) is a degenerative disease of the bone and joints, seriously affecting the quality of life of patients [1]. It often occurs in middle-aged and elderly people leading to a high disability rate. Indeed, on a global scale, there were about 303.1 million prevalent cases of hip and knee OA, with an age-standardised prevalence estimate of 3754.2 per 100,000 [2]., bringing huge economic burdens to families of affected patients and society.

OA is characterized by degeneration of the articular cartilage and secondary bone hyperplasia. The pathogenesis of joint diseases caused by mechanical, metabolic, inflammatory and immune factors remains unclear [3]. OA presentation often occurs in the knee, hip, ankle and vertebral joints with clinical manifestations characterized by slow development of joint pain, stiffness, swelling, movement limitation and deformity [4]. The incidence of cardiovascular events and all-cause mortality increase with disease progression [5].

$\mathrm{OA}$ is classified into primary and secondary OA. The appearance of primary OA mostly occurs in the middleaged and elderly, without clear induction, which is related to genetic, obesity and physical factors [6]. The appearance of secondary OA occurs in young adults, secondary to trauma, inflammation, accumulated strain or congenital diseases, and so on [7]. Currently, OAspecific biomarkers are still lacking in the clinical setting. Commonly used drugs for OA treatment include paracetamol, non-steroidal anti-inflammatory drugs (NSAI Ds), and opioids. However, NSAID and opioids display a wide variety of marked side effects, so they are not suitable for all patients. Although drugs such as corticosteroids are also commonly used in the clinic, they usually have only short-term efficacy [8]. Therefore, it is of great significance to explore the pathological mechanism of $\mathrm{OA}$ in an attempt to discover new targets for the diagnosis and treatment of OA.

Recently, studies have found that Core binding factor$\beta$ (Cbf $\beta)$ participates in chondrocyte proliferation, differentiation and osteogenesis [9]. The core binding factors are composed of the $\mathrm{Cbf} \alpha$ and $\mathrm{Cbf} \beta$ subunits: the $\alpha$ subunit is namely a Runx family (Runx) protein (including Runx1-3). Cbf $\beta$ does not directly bind DNA, but enhances the binding of Runx to DNA [10]. Cbf $\beta$ forms a complex with Runx to regulate the transcription of downstream genes, as well as the maturation and differentiation of hematopoietic stem cells [11]. The binding with Runx2 participates in the differentiation of chondrocytes and subchondral osteocytes, further affecting the development of the bone and joint [12]. Johnson et al. [13] demonstrated that target intervention to disrupt the interaction between the filament protein $\mathrm{A}$ and Cbf $\beta$ affects the gene transcription of Cbf $\beta$-Runx 1 , regulates the process of induced differentiation of chondrocytes, and may promote the repair of degenerated cartilage seen in OA. The study suggested that taking $\mathrm{Cbf} \beta$ and $\mathrm{Cbf} \beta /$ Runx as putative targets might help in the elucidation of a novel pathway for the early clinical diagnosis and treatment of OA. However, most of the current studies on $\mathrm{Cbf} \beta$ have investigated osteogenesis and chondrogenesis, and whether the abnormal expression of $\mathrm{Cbf} \beta$ has an impact on the degenerative disease of the articular cartilage remains unclear.

On the basis of clinical investigation, this study aims to further investigation and discuss the effect of abnormal expression of $\mathrm{Cbf} \beta$ on proliferation, differentiation and apoptosis of chondrocytes, as well as determining its association with articular cartilage degeneration, with the over-arching aim of providing newly discovered ideas for exploring novel target for the diagnosis, treatment and pathogenesis of OA.

\section{Methods \\ General information}

Patients with knee osteoarthritis (KOA) undergoing total knee arthroplasty in the Orthopedic Department of the Affiliated Hospital of Guizhou Medical University from July 2018 to March 2019 were enrolled to the experimental group, and those with traumatic emergency lower limb amputation were enrolled to the control group. The diagnostic criteria for KOA referred to the Guidelines for the Diagnosis and Treatment of Osteoarthritis (2018) and the AAOS Guidelines for EvidenceBased Medicine of Knee Osteoarthritis (2013) of the Orthopedic Branch of the Chinese Medical Association. The study was approved by the Ethics Committee of the Affiliated Hospital of Guizhou Medical University (Approval No. 2018 Lunshen (15)) and registered for clinical trials in ChiCTR (http://www.chictr.org.cn): ChiCTR1 800017066. The principle of voluntary participation was adopted for the collection of experimental specimens. Before collection, the subjects or family members were briefed on the study aims and rationale, and this explanation provided them an understanding of the study prior to them signing the consent document. Inclusion and exclusion criteria for study subject enrollment were as follows:

Experimental group: Inclusion criteria included the following: (1) met the diagnostic criteria for KOA in this study; (2) no history of knee joint infection or surgical trauma; (3) no history of joint cavity puncture or injection therapy; (4) no history of hormonal, immunosuppressant or other drug therapy; (5) no serious systemic diseases or obvious abnormalities in liver and kidney function. Exclusion criteria: (1) chronic consumptive diseases, such as tumors and tuberculosis; (2) other types of arthrosis diseases, such as gout and rheumatoid arthritis; 
(3) knee deformity and dysfunction caused by congenital factors; (4) patients with psychiatric diseases that were incapable of cooperating with investigators.

Control group: Inclusion criteria: (1) older than 18 years of age; (2) no symptoms such as chronic knee pain, no history of knee joint disease, trauma or surgery; (3) no history of hormonal, immunosuppressant or other drug therapy; (4) no history of serious systemic diseases or significant abnormalities in liver and kidney function. Exclusion criteria: (1) chronic consumptive diseases, such as tumor and tuberculosis; (2) special conditions such as psychiatric diseases that were incapable of cooperating with investigators; and (3) women during menstruation and pregnancy.

\section{Experimental procedure}

\section{Collection and observation of human cartilage tissue}

Specimen collection The full cartilage of the femoral condyle and tibial plateau was taken and cut into pieces on a super-clean bench for immediate primary chondrocyte culture. The remaining cartilage tissue was subpackaged into $5 \mathrm{ml}$ EP tubes: one was immersed and fixed in 4\% paraformaldehyde (Cat. No. P1110, Solarbio Science and Technology Co., Ltd., Beijing, China), and the remainder was frozen at $-80^{\circ} \mathrm{C}$.

Histological observation of cartilage specimens HE staining (Hematoxylin and Eosin staining kit, Cat. No. G1120, Solarbio Science and Technology Co., Ltd., Beijing, China): cartilage tissues were fixed in $4 \%$ paraformaldehyde for $48 \mathrm{~h}$ and decalcified with decalcification solution (G1105, Servicebio Technology Co., Ltd., Wuhan, China) for 4 wks, followed by routine paraffinembedded sectioning, hematoxylin staining for $10 \mathrm{~min}$, and rinsing with running water. Then, the sections were differentiated for $30 \mathrm{~s}$, immersed in water for $15 \mathrm{~min}$; stained with eosin for $1 \mathrm{~min}$, rinsed and immersed with running water for $2 \mathrm{~min}$, followed by dehydration, clearing, and sealing with neutral gum (Cat. No. G8590, Solarbio Science and Technology Co., Ltd., Beijing, China). The sections were observed under a standard light microscope (BX63, OLYMPUS, Japan).

Safranin O-solid green staining (modified Safranin O-solid green cartilage staining kit, Cat. No. G1371, Solarbio Science and Technology Co., Ltd., Beijing, China): the cartilage tissues were decalcified with EDTA decalcification solution (Cat. No. DD0002, Leagene Biotech Co., Ltd., Beijing, China), embedded in paraffin, sectioned, stained with safranin O-solid green, and observed under a standard light microscope (BX63, OLYMPUS, Japan).

Immunohistochemical staining of collagen type II (COL2A1 polyclonal antibody, Cat. No. bs-10589R,
Bioss, Beijing, China) was performed according to the instructions of the ready-to-use immunohistochemical Elivision $^{\text {tx }}$ super kit (Elivision ${ }^{\text {Tx }}$ super HRP Mouse/Rabbit IHC Kit, Cat. No. KIT-9922, Maixin Biotech. Co. Ltd., Fuzhou, China): the cartilage tissues were decalcified with EDTA decalcification solution, embedded in paraffin, sectioned, stained by type II collagen immunohistochemistry, and observed under a standard light microscope (BX63, OLYMPUS, Japan). The integrated optical density (IOD) of positive cells was analyzed by Image Pro-Plus 6.0 software.

Western immunoblot Western immunoblotting was used to detect the expression differences of $\operatorname{Cbf} \beta$, MMP13, IL-1 $\beta$, YKL-40, and COMP in both normal and OA cartilage tissues: protein was extracted from the cartilage tissue in liquid nitrogen, and the protein concentration was determined by the $\mathrm{BCA}$ protein assay kit (Cat. No. PC0020, Solarbio Science and Technology Co., Ltd., Beijing, China). Using the measured protein content as reference, equal amounts of protein were added to each well, and then the protein content was verified according to the reference of $\beta$-actin (Cat. No. AC026, Abclonal Biotech Co., Ltd., Beijing, China). The experiment was repeated to adjust the amount of protein loading to make consistent comparisons between samples, which were then loaded at $40 \mu \mathrm{g}$ for each protein for SDS-PAGE, followed by transmembrane blotting, and incubation with the primary antibody, and the secondary antibody respectively (HRP Goat Anti-Rabbit IgG, 1:100, Cat. No. AS014, Abclonal Biotech Co., Ltd., Beijing, China). The membrane was developed by the Bio-Rad chemiluminescent imaging system, and Image J 2X software (https://sourceforge.net/projects/ij2x/) that was used for semi-quantitative analysis of the bands to calculate relative expression. $\operatorname{Cbf} \beta$ (Cat. No. bs-6984R), MMP13 (Cat.No.bs-10250R), IL-1 $\beta$ (Cat.No.bs-20448R), YKL-40 (Cat.No.bs-10215R), and COMP (Cat.No.bs10286R) in the cartilage tissues were measured (above antibodies were all purchased from Bioss Biotechnology Co., Ltd., Beijing, China).

\section{Human chondrocyte culture}

Fresh articular cartilages were cut into pieces and washed in PBS three times, cut to $1 \mathrm{~mm}^{3}$, washed twice in PBS, digested with $0.25 \%$ trypsin for $30 \mathrm{~min}$, centrifuged at $1500 \mathrm{rpm}$ for $5 \mathrm{~min}$ to discard the supernatant, washed twice in PBS, digested with $0.2 \%$ collagenase type II in a $37^{\circ} \mathrm{C}$ water bath for $3-5 \mathrm{~h}$ until the tissue blocks basically disappeared and the digestive fluid became turbid, and then filtered with 150 mesh cell sieve to collect the filtrate at $1500 \mathrm{rpm}$ for $10 \mathrm{~min}$. The supernatant was resuspended in PBS, centrifuged again and then resuspended in 20\% FBS high-sugar DMEM culture 
medium, and then centrifuged at $1500 \mathrm{rpm}$ for $5 \mathrm{~min}$ to discard the supernatant, which was repeated twice. Finally, the cells were added to a high-sugar DMEM medium containing 20\% FBS and 1\% 100x penicillin/ streptomycin antibiotics to adjust the density to $0.5 \times$ $10^{6}-1 \times 10^{6}$ cells $/ \mathrm{ml}$, and cultured at $37^{\circ} \mathrm{C}$, in a $5 \% \mathrm{CO}_{2}$ in air atmosphere. When the adherent cells were fully confluent at the base of the culture flask, the cells were digested by $0.25 \%$ trypsin and subcultured for future use. The third generation cells were selected for subsequent experiments. Normal and OA chondrocytes were cultured respectively. Immunocytochemical staining of type II collagen showed that the cells in both groups were positive with a brown-yellow cytoplasm, and the nucleus was basically unstained, which indicated that the cells were successfully cultured with cartilage characteristics. At the same time, combined with the sampling site, the above cultured cells were confirmed to have met the needs of subsequent studies. When normal chondrocytes were passaged to the 5th cycle or OA chondrocytes to the 4th cycle of culturing, the cells lost their original morphology and had transformed into a fibroblast-like shape. Thus, the third generation of cells was used in the following studies.

\section{Construction of over-expression and inhibition of human chondrocyte Cbf $\beta$ gene}

\section{Construction of human $\mathrm{CBFB} / \mathrm{pCDH}$ lentiviral vector} PCDH cDNA Cloning and Expression Lentivectors, pCDH-MCS-T2A-copGFP-MSCV (Catalog \#: cd523a-1, System Biosciences, USA) was used to construct and obtain the $\mathrm{Cbf} \beta$ gene-overexpressed lentiviral vector plasmid. The complete CDS sequence of the human Cbf $\beta$ sequence was searched on GenBank (NCBI Reference Sequence: NM_022845.2; Homo sapiens core-binding factor beta subunit (CBFB), transcript variant 1 , mRNA; full length of $564 \mathrm{bp}$ ). The primer was designed as follows: EcoRI-hCBFB-F: CGG AAT TCA TGC CGC GCG TCG TGC CCG A; Not I-hCBFB-R: TTG CGG CCG CTT AAC GAA GTT TGA GGT CAT. PCR amplification of gene fragments: high fidelity Taq $50 \mu \mathrm{L}$ PCR: double distilled water $30.5 \mu \mathrm{L}$; upstream primer $2 \mu \mathrm{L}$; downstream primer $2 \mu \mathrm{L}$; template CDNA $2 \mu \mathrm{L}$; dNTP $4 \mu \mathrm{L}$; Mgcl2 $4 \mu \mathrm{L}$; 10 EXTaq Buffer $\left(\mathrm{Mg}^{2+}\right.$ free) $5 \mu \mathrm{L}$; and EXTaq $0.5 \mu \mathrm{L}$ in a total of $50 \mu \mathrm{L}$ in the PCR device. The program was as follows: (1) $94{ }^{\circ} \mathrm{C}, 3 \mathrm{~min}$; (2) $94{ }^{\circ} \mathrm{C}, 30 \mathrm{~s}$; (3) $52^{\circ} \mathrm{C}, 30 \mathrm{~s}$; and $\left(472{ }^{\circ} \mathrm{C}, 1 \mathrm{~min}\right.$; Go to Step 2, total 30 cycles; (5) $72{ }^{\circ} \mathrm{C}, 10 \mathrm{~min}$; Hold at $4{ }^{\circ} \mathrm{C}$. After gene fragment amplification, the $\mathrm{Cbf} \beta$ gene gel was recovered, and gene fragments and plasmid vectors were annealed. The operation was performed following the instructions found in the pCDH cDNA Cloning and Expression Lentivectors User Manual.
Double enzymatic digestion was comprised of the following: pCDH vector $3 \mu \mathrm{l}$; restriction endonuclease EcoRI $1 \mu \mathrm{l}$, NotI $1 \mu \mathrm{l} ; 10 \times$ Buffer $2 \mu \mathrm{l}$ and components composed to a volume of $20 \mu \mathrm{L}$ in ddH2O. Target gene $5 \mu \mathrm{l}$; restriction enzyme EcoRI $1 \mu \mathrm{l}$, NotI $1 \mu \mathrm{l} ; 10 \mathrm{x}$ Buffer $2 \mu \mathrm{l}$; and components composed to a volume of $20 \mu \mathrm{L}$ in $\mathrm{ddH} 2 \mathrm{O}$. The samples were mixed well and digested at $37^{\circ} \mathrm{C}$ for $30 \mathrm{~min}$. Expression vector connection: target gene $1 \mu \mathrm{l}$; PCDH-GFP $1 \mu \mathrm{l} ; 10 \mathrm{x}$ T4 Buffer $1 \mu \mathrm{l}$; and T4 DNA ligase $1 \mu \mathrm{l}$, following which, the volume was constituted to $10 \mu \mathrm{L}$ in $\mathrm{ddH} 2 \mathrm{O}$, and incubated at $16^{\circ} \mathrm{C}$ overnight.

The ligation product was transformed into Escherichia coli DH5alpha. Monoclonal Expansion Culture: the monoclone was selected and inoculated into $5 \mathrm{ml}$ liquid LB culture containing $100 \mathrm{mg} / \mathrm{ml}$ Amp resistance selection media, and shaken overnight at $37^{\circ} \mathrm{C}$. The bacterial liquid was transferred into a $7 \mathrm{ml}$ sterilized centrifuge tube and centrifuged at 10,000 rpm for $5 \mathrm{~min}$ at room temperature. Plasmids were extracted using the plasmid extraction kits (QIAprep Spin Miniprep Kit, Cat. No. 27104, Qiagen Bio Ltd., Dusseldorf, Germany). The cloned plasmid was used for PCR to identify the insertion of the target gene into the vector; the target gene band (564 bp) was visible after 1\% agarose gel electrophoresis of the plasmid targeted gere, and the positive cloned plasmid was identified by sequencing with the sequencing primers of $5^{\prime}$-GGG GTA CAG TGC AGG GGA AAG AAT-3'.

Construction of the human CBFB/pLKO.1 lentiviral vector Using the pLKO.1 - TRC Cloning Vector (Cat. No. Plasmid \#10878, Addgene, MA, USA), the Cbf $\beta$ inhibition lentiviral vector plasmid was constructed. shRNA targeting sequences were searched online through http://sirna.wi.mit.edu/, and the human $\mathrm{Cbf} \beta$ target sequence was found to be: AAG AGA AGC AGG CAA GGT ATA. According to the requirement of the pLKO.1 - TRC Cloning Vector Protocol, the hairpin structure shRNA oligonucleotide was designed and synthesized using a template as follows: human $\mathrm{CBFB}$ shRNA: 5'CCGG AAG AGA AGC AGG CAA GGT ATA CTC GAG TAT ACC TTG CCT GCT TCT CTT TTTTTG 3'; 5'AATT CAA AAA AAG AGA AGC AGG CAA GGT ATA CTC GAG TAT ACC TTG CCT GCT TCT CTT 3', which was completed by following the instructions of the pLKO.1 - TRC Cloning Vector Protocol Version 1.0, and inserting the hairpin-structure shRNA into the pLKO.1 vector.

The DNA oligonucleotide was solubilized in double distilled water to a concentration of $20 \mathrm{uM}$. The shRNA oligonucleotide template annealing and reaction system was as follows: forward oligo $5 \mathrm{ul}$; Reverse oligo $5 \mathrm{ul} ; 10$ x NEB buffer- 2 at $5 \mathrm{ul}$; dd $\mathrm{H}_{2} \mathrm{O}$ to $50 \mathrm{ul} ; 95^{\circ} \mathrm{C}$ for $4 \mathrm{~min}$, 
and cooling to room temperature. The pLKO.1 plasmid double enzyme digestion was as follows: pLKO.1 $6 \mu \mathrm{g}$; Age I $1 \mathrm{uL}$; EcoR I $1 \mathrm{uL} ; 10$ x NEB buffer $5 \mathrm{uL}$; and $\mathrm{dd}_{2} \mathrm{O}$ to $50 \mathrm{ul}$ with a temperature of $37^{\circ} \mathrm{C} 1 \mathrm{~h}$. Target fragment of the gel was recovered after $1 \%$ agarose gel electrophoresis. The annealed oligos were inserted into the pLKO.1 vector, and the reaction system was as follows: shRNA 2 ul; 10 x NEB T4 DNA ligase buffer 2 ul; pLKO.1 vector $20 \mathrm{ng}$; NEB T4 DNA ligase $1 \mathrm{ul}$; and $\mathrm{ddH}_{2} \mathrm{O}$ to $20 \mathrm{ul}$, which was maintained at $16^{\circ} \mathrm{C}$ overnight. After recombination transformation and monoclonal culture, an expanded culture was conducted to extract the plasmid (QIAprep Spin Miniprep Kit, Cat. No. 27104, Qiagen Bio Ltd., Dusseldorf, Germany). The next procedure was cloning plasmid double enzyme digestion to identify the correct insertion of shRNA into the vector: cloned plasmid $1 \mu \mathrm{g}$; EcoR I $0.8 \mathrm{uL}$; Nco I 0.8 $\mathrm{uL}$; $10 \times$ buffer $2 \mathrm{uL}$; and $\mathrm{ddH}_{2} \mathrm{O}$ to $20 \mathrm{uL}$; and a temperature of $37^{\circ} \mathrm{C}$, for $1 \mathrm{~h}$. Finally, $1 \%$ agarose gel electrophoresis was conducted on the reaction products, from which two fragments $(2 \mathrm{~kb}$ and $5 \mathrm{~kb})$ were shown. The positive cloned plasmids were sequenced using the primer: 5'-CAA GGC TGT TAG AGA GAT AAT TGGA-3'.

Plasmid transfection After the plasmids of the human $\mathrm{CBFB} / \mathrm{pCDH}$ and $\mathrm{CBFB} / \mathrm{pLKO} .1$ lentivirus were obtained, the OA chondrocytes were transfected with the plasmids. Cells were digested and counted 1 day before transfection, and cultured in a 6-well plate for transfection at a density of $80 \%$. Mixing ratio of transfection reagent and plasmid mass $=4 \mu \mathrm{l}: 2.5 \mu \mathrm{g}$, and then $125 \mu \mathrm{l}$ DMEM was added to each well, as well as $2.5 \mu$ g plasmid DNA, and $4 \mu \mathrm{l} \mathrm{Lipo8000}{ }^{\mathrm{mm}}$ transfection reagent (Cat. No. C0533, Beyotime Biotech Co., Ltd., Shanghai, China). After premixing in the sterile centrifuge tube, the solution was added to each well, and cultured for $48 \mathrm{~h}$ for use.

Detection of human chondrocyte proliferation and apoptosis The chondrocytes were divided into four groups: control group (normal chondrocytes), OA group (OA chondrocytes), $\mathrm{CBFB} / \mathrm{pCDH}$ group (transfection with $\mathrm{CBFB} / \mathrm{pCDH}$ ), and the $\mathrm{CBFB} / \mathrm{pLKO} .1$ group (transfection with CBFB/pLKO.1) according to the previously decribed experiment, followed by experiments of chondrocyte proliferation and apoptosis.

CCK-8 detection: cells in each group were cultured in a 96-well plate for $24 \mathrm{~h}$, and CCK- 8 cell counting Kit (cat. No. a311-01, Vazyme Biotechnology Co., Ltd., Nanjing, China) was used for detection. Absorbance values were measured at $450 \mathrm{~nm}$ by a microplate reader.

Annexin V/PI flow cytometry apoptosis (CyFlow ${ }^{\circ}$ Space; SysmexPartec GmbH, Görlitz, Germany): after trypsin digestion and collection of cells in each group by centrifugation, the experiment was conducted following the annexin V-FITC apoptosis detection kit (Cat. No. c1062l, Beyotime Institute of Biotechnology, Shanghai, China) operation manual guidelines.

Effect of CBF $\beta$ gene over-expression and inhibition on the expression of OA-related inflammatory markers and cartilage metabolism markers.

Real-time PCR detection The total RNA of chondrocytes in each group was extracted and synthesized by cDNA, based on the instruction of the hiscript II Q select RT Supermix for qPCR (+ gDNA wiper) reverse transcription Kit (Cat.no. r233-01, Vazyme Biotechnology Co., Ltd., Nanjing, China). The target gene CDS sequence was searched against the GenBank gene database, and real-time PCR primer sequence as follows:: Human CBFB forward: 5'-TTT GAA GGC TCC CAT GAT TC-3', reverse: 5' ${ }^{\prime}$-ATC TTC AAA TTC GCG TGT CC-3'; Human COMP forward: 5'- AGG ACA ACT GCG TGA CTG TG-3', reverse: 5' - GTG TCC TTT TGG TCG TCG TT-3'; Human MMP13 forward: 5'-TTG AGC TGG ACT CAT TGT CG-3', reverse: 5' GGA GCC TCT CAG TCA TGG AG-3'; Human IL-1 $\beta$ forward:5'-GGG CCT CAA GGA AAA GAA TC-3', reverse: TTC TGC TTG AGA GGT GCT GA-3'; Human YKL-40 forward: 5' -TCA AGA ACA GGA ACC CCA AC-3', reverse: $5^{\prime}$-AAA TTC GGC CTT CAT TTC CT3'; Human GAPDH forward: 5'-GAC ATC AAG AAG GTG GTG AAG CAG-3', reverse: 5'-GTC AAA GGT GGA GGA GTG GGT-3'.

Relative expression was detected by fluorescence quantitative PCR, following the instructions of the $\mathrm{AceQ}^{\circ}$ qPCR SYBR Green qPCR master mix (Cat. No. Q51101, Vazyme Biotech Co. Ltd., Nanjing, China). The primer was diluted into $5 \mu \mathrm{M}$, and mixed with the following agents in order: $10 \mu \mathrm{l}$ AceQ $^{\circ}$ qPCR SYBR Green Master Mix, $0.4 \mu$ l Forward Primer $(5 \mu \mathrm{M}), 0.4 \mu \mathrm{l}$ Reverse Primer $(5 \mu \mathrm{M}), 0.4 \mu \mathrm{l}$ ROX Reference Dye, Distilled Water (dH2O), which was added to $20 \mu \mathrm{l}$, and then amplification on the ABI Prism 7300HT (Real Time PCR amplifier) using two-step PCR. The thermo-cycling conditions constituted of the following: initial denaturation at $95^{\circ} \mathrm{C}$ for $10 \mathrm{~min}$ ( 1 cycle); denaturation at $95^{\circ} \mathrm{C}$ for $15 \mathrm{~s}$, and annealing/extension for $60^{\circ} \mathrm{C}$ for $60 \mathrm{~s}$ ( 40 cycles). DataAssist $^{\text {tix }}$ v3.0 Software (ABI) was used to analyze the results based on the $2^{-\Delta \Delta \mathrm{Ct}}$ method.

Western Immunoblotting Total protein extraction in each group was conducted as follows: cells were washed three times in PBS, lyzed with 200 ul PMSF RIPA lysate (Cat.No. P0013B, Beyotime Biotech Co., Ltd., Shanghai, China), cooled on an ice-bath for $30 \mathrm{~min}$, and then centrifuged at $12,000 \mathrm{rpm}$ at $4{ }^{\circ} \mathrm{C}$ for $5 \mathrm{~min}$. The protein 
concentration was determined by the BCAassay. The protein supernatant of different groups was collected, and denatured with $5 \times$ SDS loading buffer $(20 \% \mathrm{v} / \mathrm{v})$ and subjected to a boiling water treatment for $10 \mathrm{~min}$. SDS-PAGE was performed at $80 \mathrm{~V}$ concentrated gel voltage and $140 \mathrm{~V}$ separated gel voltage, followed by PVDF membrane transfer, and then blocking against nonspecific antibody binding activity in 5\% skimmed milk powder TBS-T solution overnight at $4{ }^{\circ} \mathrm{C}$. The membrane was further incubated with primary antibody diluted with TBS-T containing 5\% skimmed milk powder (1: 1000) at 4) (11r (11wder (the detection target was Cbf $\beta$, IL-1 $\beta$, YKL-40, COMP and MMP13 using $\beta$-actin as a reference (all the antibodies used were the same as those described previously above). The membrane was further incubated with a secondary antibody that was diluted in TBS-T containing 5\% skimmed milk powder (HRP Goat Anti-Rabbit IgG, 1:5000, Cat.No. AS014, Abclonal Biotech Co., Ltd., Beijing, China) at room temperature for $60 \mathrm{~min}$, and then developed by ECL (WBKLS0100, Millipore, USA), and photographed by Bio-Rad and recorded.

\section{Statistical method}

SPSS version 25.0 software (IBM SPSS statistics $25.0 \times 64$ ) was used for statistical analysis. Measurement data were expressed as the mean $\pm \mathrm{SD}$. The Student's t-test was used to compare both groups. One-way ANOVA followed by Tukey's post hoc test or repeated measures ANOVA was used to determine the significance of the differences between groups. The Chi square test was used for comparisons of clinical count data. An alpha value of $P<0.05$ was regarded as a statistically significant difference.

\section{Results}

\section{Study subject inclusion, cartilage acquisition and} histological observation

In this study, $53 \mathrm{KOA}$ patients were first selected for total knee arthroplasty, and 22 patients were finally included into the experimental group (obtaining the pathological cartilage tissue) and strictly following the exclusion criteria. There were 9 men and 13 women, with a mean age of $64.09 \pm 4.20$ years and an average BMI of $25.73 \pm 3.84$. The K-L grade of the knee joint was determined as being grade IV [14]. According to the inclusion criteria, 8 patients were included in the emergency trauma amputation group as the control group (obtaining normal cartilage tissue), which included 4 men and 4 women, with a mean age of $53.13 \pm 13.53$ years and a BMI of $22.69 \pm 2.71$. The K-L grade of the knee joint was 0 [14]. The age $(F=13.145, p=0.001)$ difference for both groups was significantly different $(p<$ $0.05)$, while the BMI showed no difference at all $(F=$ 1.40, $p=0.247$ ) while the gender composition also showed no different $(\chi 2=0.197, p=0.698 ; P>0.05)$ (Table 1).

The macroscopic structure of the normal cartilage in the control group (Fig. 1a) and the OA diseased cartilage in the experimental group (Fig. 1b) were significantly different. The collected cartilage of the knee joint in the control group showed a milky white appearance with a smooth and shiny surface but with no evidence of cracks, erosion or ulcer formation. In the experimental group, the specimens showed a yellow and dark color, with uneven surfaces, fissures, thin cartilage, and exposed subchondral bone. By HE staining, safranin O staining and type II collagen immunohistochemical staining, we examined the morphology and appearance of collagen as a key component of the cartilage matrix (mainly as type II collagen). The staining results showed that when compared with normal cartilage (Fig. 1a and b), the expression of the type II collagen protein in the OA cartilage was significantly decreased $(p<0.05)$. It can be seen that the normal phenotype of chondrocytes in the OA diseased cartilage persisted; however, the expression of type II collagen had decreased, and the overall structure of cartilage tissue was disordered. HE staining showed that the nuclei of the normal cartilage (Fig. 1e and f) were dark blue, the cytoplasm and cartilage matrix were pink, and demonstrated uniform staining. The cells on the superficial layer were small and round, and were arranged parallel to the cartilage surface. The cells in the deep layer were larger and oval or round. In the experimental group (Fig. $1 \mathrm{~g}$ and $\mathrm{h}$ ), the structure was disordered. There were many empty cartilage lacunae with a disordered cellular arrangement. Chondrocyte necrosis was observed in the superficial and deep layers, and the number was significantly reduced. Safranin O solid green staining showed that the matrix of the normal cartilage (Fig. 1i and $j$ ) was uniformly stained red, and the subchondral bone was green, with a clear boundary between the cartilage and subchondral bone. The OA structure of cartilage (Fig. 1k and $\mathrm{l}$ ) was disordered, the staining of the cartilage matrix was lighter, and the range of red staining was minimal, suggesting that the diseased cartilage matrix was destroyed and reduced.

\section{Expression of $\mathrm{Cbf} \beta$ and $\mathrm{OA}$ related inflammatory factors and cartilage metabolic markers in $O A$ cartilage tissue} $\mathrm{Cbf} \beta$ was highly expressed in human OA diseased cartilage tissues. In addition, the expression of inflammatory factors and cartilage metabolites, including MMP-13, IL-

Table 1 Comparison in general data

\begin{tabular}{llll}
\hline & Experimental group & Control group & $\boldsymbol{p}$ \\
\hline Age & $64.09 \pm 4.20$ & $53.13 \pm 13.53$ & 0.001 \\
BMl & $25.73 \pm 3.84$ & $22.69 \pm 2.71$ & 0.247 \\
Gender (male/female) & $9 / 13$ & $4 / 4$ & 0.698 \\
\hline
\end{tabular}




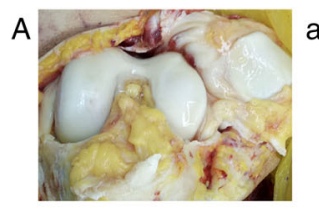

B
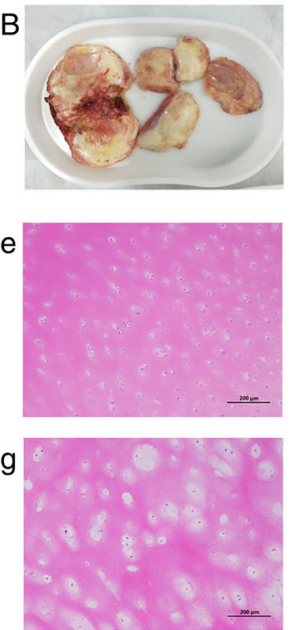

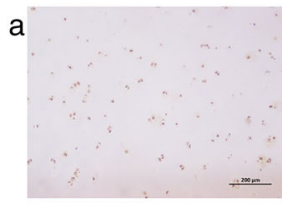

c
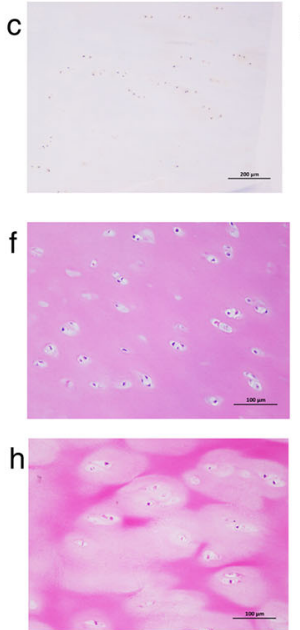

b

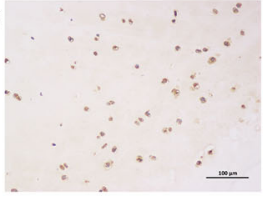

d
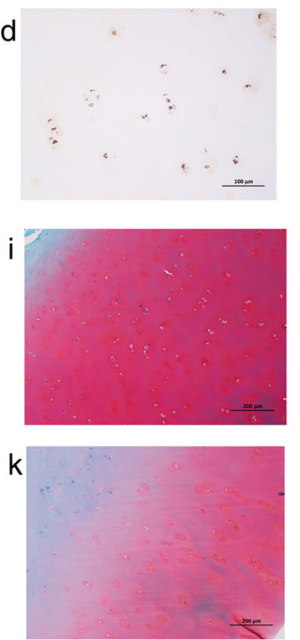
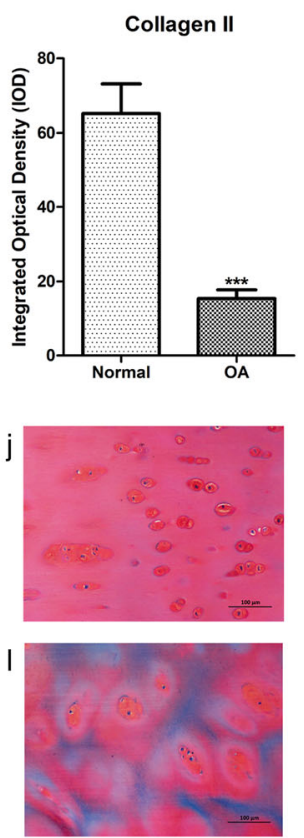

Fig. 1 Normal human knee joint cartilage (A) and OA diseased cartilage (B) macroscopic tissue structure; Collagen type II immunohistochemical staining: normal cartilage (a-100x, scale bar $=200 \mu \mathrm{m} ; \mathrm{b}-200 \mathrm{x}$, scale bar $=100 \mu \mathrm{m})$ and OA cartilage $(c-100 x$, scale bar $=200 \mu \mathrm{m} ; \mathrm{d}-200, \mathrm{xscale}$ bar $=$ $100 \mu \mathrm{m})$; HE staining: normal cartilage (e-100x, scale bar $=200 \mu \mathrm{m}$; f-200x, scale bar $=100 \mu \mathrm{m})$ and OA cartilage $(\mathrm{g}-100 x$, scale bar $=200 \mu \mathrm{m}$; h-200x, scale bar $=100 \mu \mathrm{m})$; Safranin O-solid green staining: normal cartilage $(\mathrm{i}-100 \mathrm{x}$, scale bar $=200 \mu \mathrm{m}$; j-200x, scale bar $=100 \mu \mathrm{m})$ and OA cartilage $(\mathrm{k}-$ $100 x$, scale bar $=200 \mu \mathrm{m} ; 1-200 x$, scale bar $=100 \mu \mathrm{m}$ )

$1 \beta$, COMP, and YKL-40 that were closely related to OA, were higher than those found in normal cartilage tissues. It was found that the expression of each marker was significantly different when comparing the $\mathrm{OA}$ with the control group $(p<0.001$; Fig. 2$)$.

\section{Effect of $\mathrm{Cbf} \beta$ gene over-expression and inhibition on human $\mathrm{OA}$ chondrocyte growth}

After sequence identification, the human $\mathrm{CBFB} / \mathrm{pCDH}$ lentiviral vector (Fig. 3a) and the human CBFB/pLKO.1 lentiviral vector (Fig. $3 \mathrm{~b}$ ) were effectively constructed. Viral plasmids were transfected into OA lesional chondrocytes. It was determined that $\mathrm{Cbf} \beta$ over-expression and inhibition occurred significantly in both groups (Fig. 3c). The result of CCK- 8 analysis (Fig. 3d) of the four groups indicated that abnormal expression of $\mathrm{Cbf} \beta$ had a significant negative effect on the proliferation and growth of human articular chondrocytes. Compared with the control group, the proliferation of cells in the OA group decreased significantly; moreover, the overexpression of $\mathrm{Cbf} \beta$ decreased cell viability, and the inhibition of $\mathrm{Cbf} \beta$ significantly increased cell proliferation. This indicated that the increased expression of $\mathrm{Cbf} \beta$ inhibited cell proliferation; however, the inhibition of $\mathrm{Cbf} \beta$ gene expression had an opposing effect. The results of Annexin V/PI flow cytometry apoptosis (Fig. 3e and f) showed that the apoptotic rate $(\mathrm{Q} 2+\mathrm{Q} 4)$ in the OA and
CBFB/ pCDH group with an abnormally increased Cbfßexpression was significantly increased as compared the control group $(p<0.001)$, but that of the CBFB/ pLKO.1 group was significantly down-regulated $(p<$ 0.001 ), which suggested that Cbf $\beta$ inhibition effectively reduce early and late apoptosis (Q4), and number of necrotic $(\mathrm{Q} 2)$ OA chondrocytes.

\section{Effects of Cbf $\beta$ over-expression/inhibition on the expression of $O A$ related inflammatory factors and cartilage metabolic markers}

Real-time PCR (Fig. 4a) and Western blot (Fig. 4b) were used to detect inflammation-related factors and marker products (i.e., IL-1beta, MMP-13, COMP, YKL-40) of cartilage metabolic abnormalities closely related to $\mathrm{OA}$ in the control group, $\mathrm{OA}$ group, $\mathrm{CBFB} / \mathrm{pCDH}$ group, and the $\mathrm{CBFB} / \mathrm{pLKO} .1$ group. Regulating the expression of $\mathrm{Cbf} \beta$ in chondrocytes induced changes in expression of the four OA markers (IL-1beta, MMP-13, COMP, YKL-40). Real-time PCR results showed that (Fig. 4a) mRNA expression of $\mathrm{Cbf} \beta$ and other related markers were all increased in the OA group as compared with the control group, and there were significant differences in the observed increases in COMP, MMP13 and YKL$40(p<0.05)$. Compared with the OA group, the mRNA expression levels of MMP13 and IL-1 $\beta$ in OA cells were significantly increased when $\mathrm{Cbf} \beta$ was over-expressed 


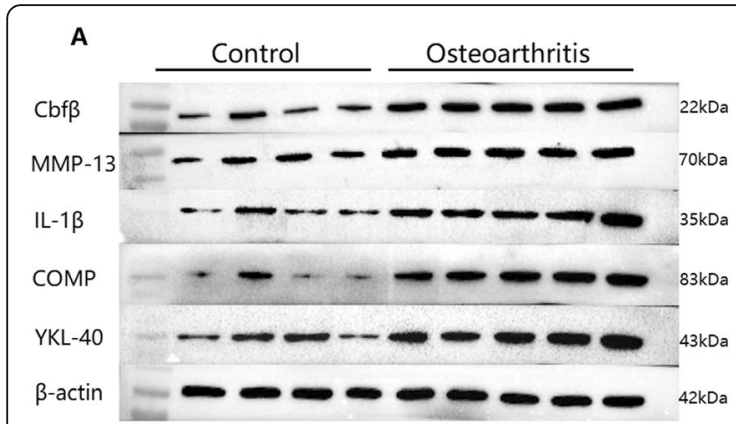

B

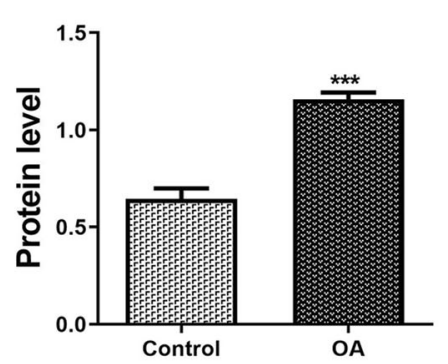

C

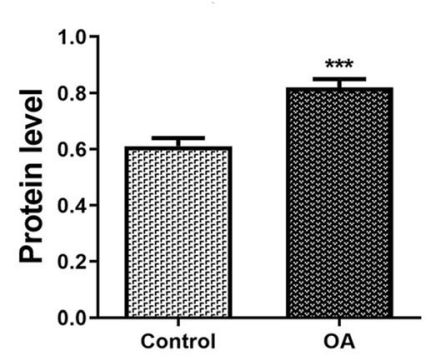

D

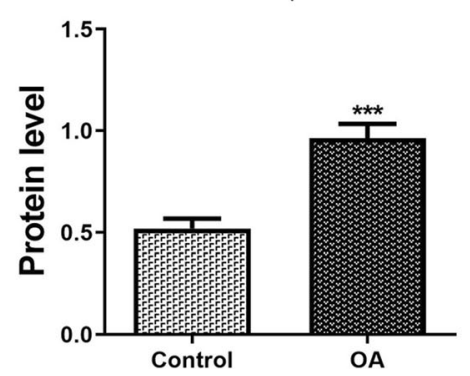

COMP

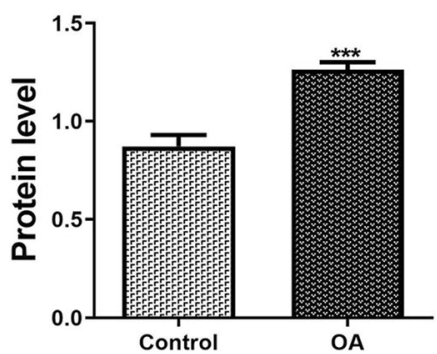

YKL-40

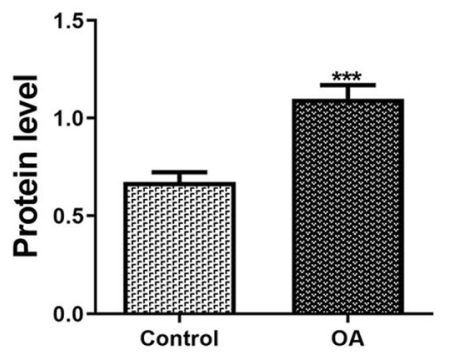

Fig. 2 Western blot analysis of human cartilage tissue, A: Western blot band map of each protein, B-F: quantitative histogram of protein bands for the expression of Cbf $\beta, M M P-13, I L-1 \beta, C O M P$, and YKL-40 (***: $p<0.001)$

$(p<0.05)$, and the expression of COMP and YKL-40 was somewhat up-regulated. Compared with the OA group, COMP, MMP13 and YKL-40 mRNA expression in chondrocytes was also significantly decreased after inhibition of Cbf $\beta$ expression $(p<0.05)$. Western blot results showed that when compared with the control group, protein expression of $\mathrm{Cbf} \beta$ and other related markers were significantly increased in the OA group $(p<0.01)$. When compared with the OA group, the expression of other cellular markers showed an increasing trend when $\mathrm{Cbf} \beta$ was over-expressed, and the expression of COMP, MMP13, IL-1 $\beta$ and YKL-40 in chondrocytes was significantly decreased when $\mathrm{Cbf} \beta$ expression was inhibited $(p<0.001)$.

\section{Discussion}

Joint degenerative diseases, such as osteoarthritis are closely related to the functional changes of chondrocytes in the articular cartilage tissue [15]. Abnormal cell proliferation, differentiation and apoptosis leads to cartilage matrix degeneration and calcification, which indicates that the pathogenesis of osteoarthritis is closely related to the proliferation, differentiation and death of chondrocytes. Studies have confirmed that multiple OArelated changes in cytokines and molecular signals can induce chondrocyte abnormalities [16-19], which indicates that studying the mechanism of chondrocyte degeneration and apoptotic abnormalities are promising approaches to help guide early diagnosis and treatment protocols for osteoarthritis in the clinic. At the same time, development of specific biological and drug preparations for chondrocyte proliferation, differentiation and apoptosis mechanisms is expected to effectively delay the development of the degeneration of the OA articular cartilage [15].

Recently, it has been found that core binding factor $\beta$-subunit $(\mathrm{Cbf} \beta)$ is closely related to chondrocyte differentiation and osteogenesis [9]. Cbf $\beta$ controls the balance between chondrocyte proliferation and differentiation, and promotes chondrocyte proliferation and osteoblast differentiation by up-regulating Ihh expression, and inhibits PPR expression to enhance chondrocyte differentiation [20]. Miller et al. [21] established a transgenic model of $\mathrm{Cbf} \beta$ mice, in which the promoter and enhancer of the Tek gene were used to express the fusion proteins of GFP and $\mathrm{Cbf} \beta$ in the mice with $\operatorname{Cbf} \beta$ defects. The mice died due to severe abnormal bone development after birth. Simultaneously, it has been confirmed that [12] mice with abnormal $\mathrm{Cbf} \beta$ expression died one day after birth. Due to abnormal development of chondrocytes, both intramembranous and endochondral osteogenesis were significantly inhibited. However, the relationship between abnormal expression of $\mathrm{Cbf} \beta$ and degenerative 


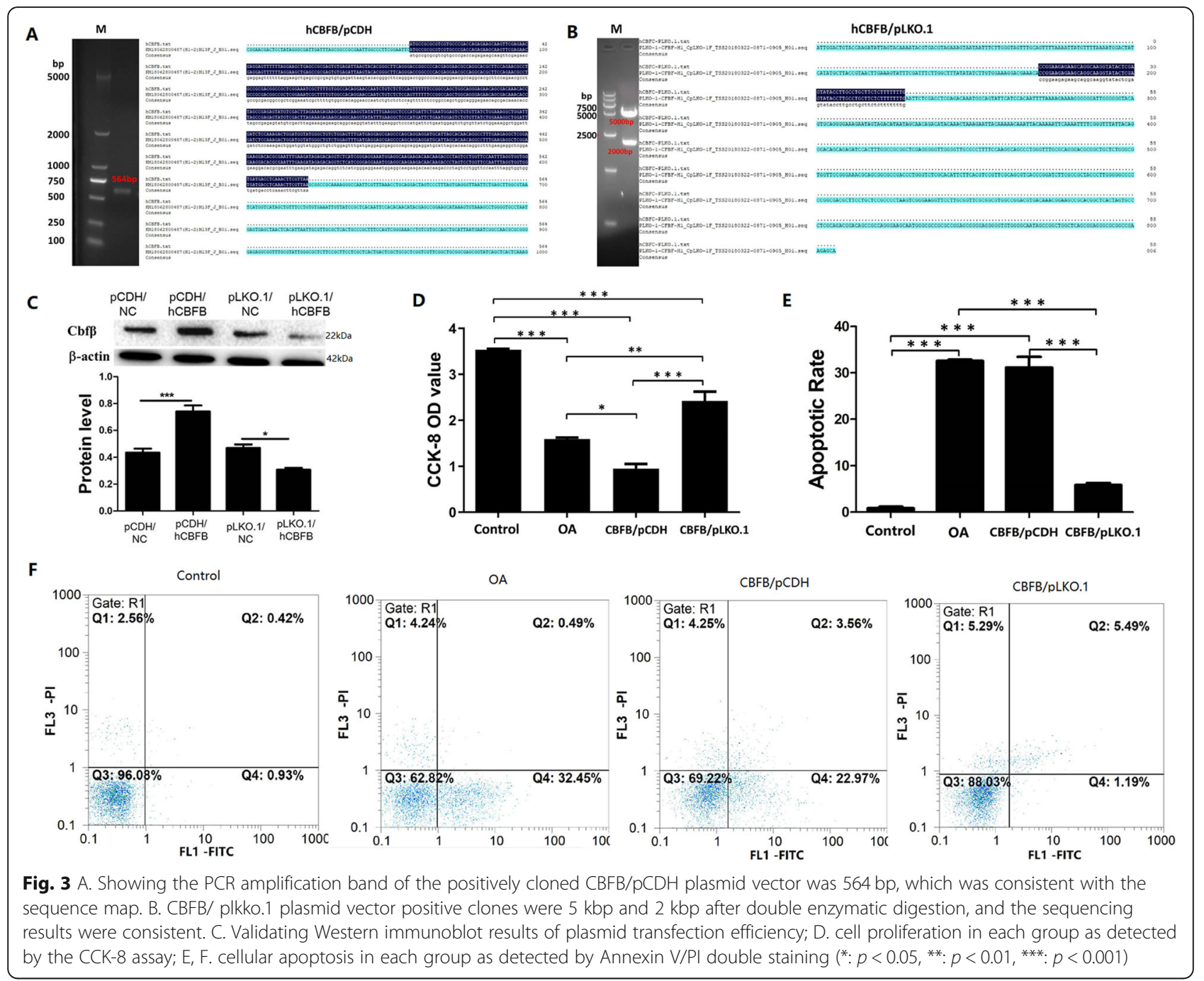

diseases like OA has not been clearly clarified up to now.

In this study, biomarkers such as OA-related cartilage metabolites and inflammatory factors were detected in the cartilage. The results showed that $\mathrm{Cbf} \beta$ was highly expressed in human OA diseased cartilage. Meanwhile, the expression of protein markers to include IL-1 $\beta$, MMP-13, comp and YKL-40 that were closely related to OA were significantly higher than that seen for normal cartilage.

Cartilage is composed of chondrocytes and extracellular matrix (ECM). Chondrocytes synthesize and secrete matrix components. In the inflammatory condition and during OA progression, significant changes in matrix degradation proteins provokes ECM remodeling. Changes in ECM will affect the chondrocyte microenvironment, which will further promote OA progression under inflammatory condition. Many studies have shown that functional changes in cartilage caused by proliferation, differentiation and apoptosis of chondrocytes are related to the degenerative joint diseases. Abnormal apoptosis often leads to degeneration and calcification of the cartilage matrix [15]. As shown in Fig. 1, when compared with the normal diseased OA cartilage, the color was obviously dull, and the surface was not smooth with many cracks, and the cartilage was inelastic with uneven thickness, and even some joint areas were obviously degenerated and missing. Histological section staining showed that the cartilage structure level was disordered, and displayed more empty cartilage lacunae with a disordered cellular arrangement. In addition, normal cells were decreased significantly in both the surface and deep layer, the destruction of the cartilage ECM was obvious, and the expression of type II collagen in tissues was significantly decreased.

It is widely known that there is a "silent period" in the onset of osteoarthritis, in which patients may not have any clinical symptoms, nor obvious abnormalities by imaging examination. However, cartilage tissues might display extensive metabolic changes [22]. Cartilage 
A
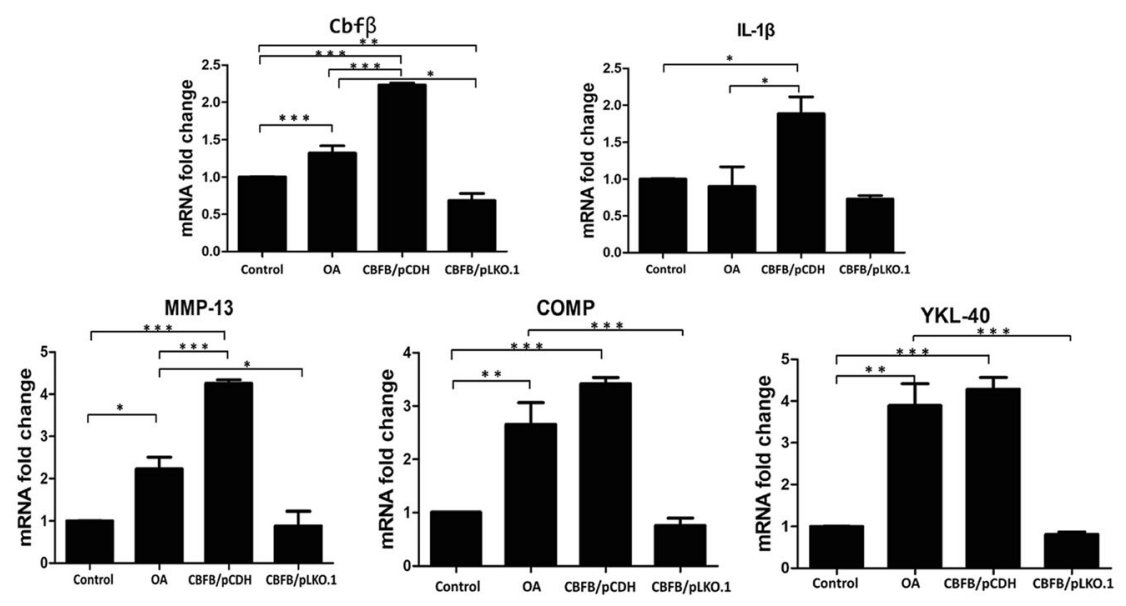

B
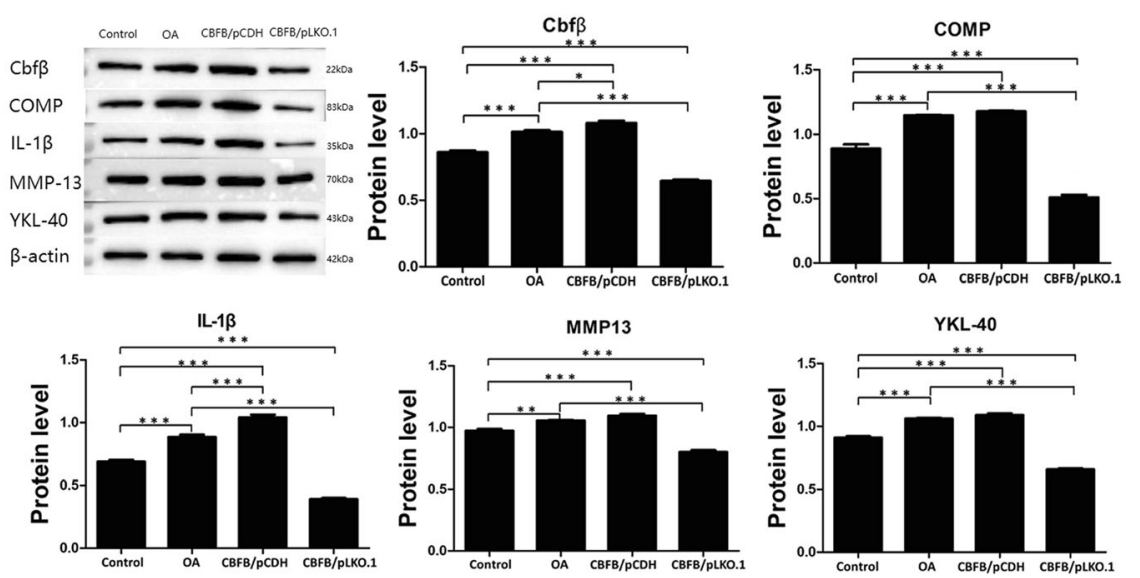

Fig. 4 A. Statistical histogram of real-time PCR results, B. Bands and statistical histograms of Western blot results $\left({ }^{*} p<0.05,{ }^{* *} p<0.01\right.$, $\left.{ }^{* * *} p<0.001\right)$

destruction and local inflammatory response are key pathological changes seen in OA, and thus biomarkers associated with $\mathrm{OA}$ are mainly associated with cartilage metabolites and inflammatory factors [23]. In the process of cartilage degeneration, COMP, YKL-40, KS, CTX-II, PIICP and other small molecular products of cartilage matrix degradation are released gradually, and change with progression of the disease [22]. Verma et al. [24] confirmed that the synthesis and degradation of COMP is closely related to cartilage metabolism. YKL40 [25] expression levels indirectly indicate joint destruction, which can be used to evaluate the degree of joint degeneration/destruction.

Local inflammatory responses are also one of the most important pathological changes in OA development. Inflammatory mediators break the dynamic balance of cartilage matrix metabolism by affecting the metabolic activity of normal chondrocytes, which accelerates cartilage destruction [26]. The inflammatory markers, especially interleukins [27] (ILs) and matrix metalloproteinases [28-30] (MMPs) have been widely investigated. In previous studies, IL-1 $\beta$ has been shown to play a key role in the development and progression of OA [31,32]. The results of Özler $\mathrm{K}$ et al. [33] showed that MMP-13 expression in the joint fluid of patients with severe KOA was significantly related to the WOMAC score. As a specific biomarker of OA, MMP-13 could be used to determine the extent of cartilage damage to evaluate disease severity.

This is also concordant with the results of our previous detection assays on the pathological cartilage. Combined with the results of previous studies, $\mathrm{Cbf} \beta$ was selected as the main target, as well as inflammatory related factors and cartilage metabolites including IL-1 $\beta$, MMP-13, COMP and YKL-40, as the reference for chondrocyte abnormality and degeneration in the later stage of the disease. As a chaperonin of the Runx family of transcription factors, Cbf $\beta$ participates in cellular metabolic processes, such as osteogenesis and chondrogenesis, and plays an important role in chondrogenesis $[9,12,34]$. Based on previous conclusions, it was speculated that $\mathrm{Cbf} \beta$ might play an important role in chondrocyte apoptosis and cartilage tissue degeneration in 
OA pathogenesis, but the specific role remained to be elucidated.

In this study, a significant abnormal increase in $\mathrm{Cbf} \beta$ was found in diseased cartilage tissue of OA patients and abnormal changes in various cartilage metabolites and inflammatory factors closely related to OA disease. Thus, it was speculated that abnormally increased expression of $\mathrm{Cbf} \beta$ in the cartilage tissue of osteoarthritis might play an important regulatory role upstream of the pathogenic pathway. To further clarify the effect, after culturing normal and OA chondrocytes in vitro, we performed $\mathrm{Cbf} \beta$ gene over-expression and inhibition treatment on OA-diseased chondrocytes by lentiviral transfection. Finally, two phenotypic chondrocytes, $\mathrm{CBFB} / \mathrm{pCDH}$ (Cbf $\beta$ over-expression) and CBFB/pLKO.1 (Cbf $\beta$ expression inhibition), were obtained. The results of CCK8 cell proliferation showed that the in vitro proliferation of cells was significantly enhanced after expression of CBFB/pLKO.1 was inhibited $(p<0.01)$. However, after further over-expression of $\mathrm{Cbf} \beta$ in diseased chondrocytes, the proliferation in the $\mathrm{CBFB} / \mathrm{pCDH}$ group had further declined. The proliferation of normal chondrocytes was higher from that of the other three groups in CCK8 assays. However, the in vitro cell studies showed that inhibiting and regulating $\mathrm{Cbf} \beta$ expression in OA diseased cells effectively improved the proliferative activity of chondrocytes. Annexin V/PI flow cytometry also confirmed that after the inhibition of $\mathrm{Cbf} \beta$ gene expression in the CBFB/pLKO.1 group, the viable cell density was significantly increased from 62.82 to $88.03 \%$, and that of early apoptosis was significantly decreased from 32.45 to $1.19 \%$.

Our study indicated that selective up-regulation of $\mathrm{Cbf} \beta$ expression in chondrocytes was associated with a significant increase in the total apoptotic rate, and the cell proliferative activity was also reduced. However, inhibition of $\mathrm{Cbf} \beta$ expression could regulate and reduce the total apoptotic rate, and significantly increase the cell proliferative activity, indicating that $\mathrm{Cbf} \beta$ might be closely related to pathological manifestations, such as abnormal chondrocyte apoptosis and cartilage tissue degeneration. Indeed, the apoptotic results of the Annexin V/PI flow cytometry assay demonstrated that after overexpression of $\mathrm{Cbf} \beta$ in the diseased chondrocytes of the $\mathrm{CBFB} / \mathrm{pCDH}$ group, the viable cell density, the total apoptotic rate and the general expression of chondrocytes in OA lesions were quite similar. While, the count of living cells and the total apoptotic rate in the CBFB/ pLKO.1 group had also significantly improvement. Will it also affect the expression of OA-related inflammatory factors and cartilage metabolites in cells? To answer that question, the expression of OA-related inflammatory factors and cartilage metabolites in chondrocytes of each group was analyzed at both the gene and protein levels.
The results of real-time PCR and Western blotting showed that there was also a certain expression level of Cbf $\beta$, IL-1 $\beta$, MMP-13, COMP and YKL-40 in normal chondrocytes of the control group (Fig. 4); however, there was a significant increase in $\mathrm{Cbf} \beta$ in the OA group $(p<0.001)$. Simultaneously, the expression of the above inflammatory factors and cartilage metabolites were all increased - at least to different extents, which was consistent with our findings.

In the study, we also processed OA chondrocytes by over-expression and inhibition of $\mathrm{Cbf} \beta$, which were then divided into the $\mathrm{CBFB} / \mathrm{pCDH}$ group $(\mathrm{Cbf} \beta$ overexpression) and the CBFB/pLKO.1 group (Cbf $\beta$ expression inhibition). The expression of COMP and YKL-40 in the $\mathrm{CBFB} / \mathrm{pCDH}$ group showed slight increases in detection by real-time PCR and Western immunoblot analysis. Although there was no significant statistical difference with the OA group $(p>0.05)$, the COMP and YKL-40 expression in the $\mathrm{CBFB} / \mathrm{pCDH}$ and the $\mathrm{OA}$ group were significantly higher than was found in normal cells $(p<0.01)$. Studies have shown that COMP [24] and YKL-40 [25] metabolites were mainly produced by chondrocytes, and were abnormally increased in chondrocyte degeneration or the inflammatory process, which can be used as diagnostic indices for articular cartilage degeneration and destruction.

The above results indicated that an abnormal increase in $\mathrm{Cbf} \beta$ in chondrocytes significantly affected the apoptosis rate and proliferative activity of cells, and caused an abnormal increase in OA-related cartilage metabolites and inflammatory factors, which was consistent with the characteristics of Annexin V/PI flow cytometry apoptosis assay in this study. In the CBFB/pLKO.1 group, with significantly inhibited $\mathrm{Cbf} \beta$ expression, the protein expression of disease-related inflammatory factors and cartilage metabolites was significantly decreased as compared with those of the OA group $(p<0.001)$. The result of the Annexin V/PI flow cytometry and CCK-8 detection assays in this study indicated that in the pathological mechanism of OA development, $\mathrm{Cbf} \beta$ played an upstream regulatory role in the inflammatory response and abnormal apoptosis signaling pathway. The abnormal expression of $\operatorname{Cbf} \beta$ might be a key factor that induces the inflammatory response and abnormal apoptosis of chondrocytes.

Cbf $\beta$ plays a regulatory role in the dynamic balance between chondrocyte proliferation and differentiation [20]. As a co-transcription factor of Runx2 [35, 36], Cbf $\beta$ forms a heterodimer with the Runx2 protein, playing an important positive regulatory role in chondrocyte differentiation and osteogenesis [37]. Thus, abnormal expression of $\mathrm{Cbf} \beta$ is closely related to the apoptotic changes of articular chondrocytes and cartilage tissue degeneration. In the study, vector virus was used to regulate $\mathrm{Cbf} \beta$ in human diseased chondrocytes in vitro, demonstrating a significant effect of $\mathrm{Cbf} \beta$ regulatory changes 
on the proliferation, differentiation and apoptosis of chondrocytes. The inhibition of $\mathrm{Cbf} \beta$ expression significantly reduced OA-related biomarkers to include IL-1 $\beta$, MMP-13, COMP, and YKL-40. Moreover, the abnormal proliferation and apoptosis of OA-diseased chondrocytes was improved. Thus, the inhibition of $\mathrm{Cbf} \beta$ expression might play a role in delaying cartilage degeneration, which was expected to provide a novel pathway for the exploration of new targets for the diagnosis, treatment, and pathogenesis of OA.

\section{Conclusions}

In this study, there was a significant abnormal increase in $\mathrm{Cbf} \beta$ in the articular cartilage of OA patients. Simultaneously, the expression of inflammatory factors and cartilage metabolites closely related to $\mathrm{OA}$ was also significantly increased, indicating that the expression changes in $\mathrm{Cbf} \beta$ was closely related to the occurrence and development of human cartilage degeneration. Our study provides a valuable clinical investigation and laboratory data for the study of OA pathogenesis; however, it has some limitations due to the limited clinical sample size. Future studies will need multi-center clinical trials with large sample sizes. By expanding the sample size of clinical studies and the diversity of included samples, and further connecting with more upstream multi-level regulatory gene expression studies on circRNA, lncRNA, miRNA and other cellular and molecular levels, it will be helpful to clarify the important role of $\mathrm{Cbf} \beta$ and related markers in osteoarthritis pathogenesis.

\section{Abbreviations}

OA: Osteoarthritis; NSAIDs: Non-steroidal anti-inflammatory drugs; KOA: Knee osteoarthritis; Cbf $\beta$ : Core binding factor $\beta$-subunit; ECM: Extracellular matrix; ILs: Interleukins; MMPs: Matrix metalloproteinases; MMP-13: Matrix metalloproteinase-13

\section{Acknowledgements}

We should like to thank Dr. Yu Li and her team from the Biochemistry and Molecular Biology of Yunnan University, for their assistance in the relevant experimental techniques.

\section{Authors' contributions \\ Guangdi Li conceived and coordinated the study, designed, performed and analyzed the experiments, wrote the paper. Mi Zhang, Yuan Huang, Jiafei Yang, Lianghong Dong, Hao Shi, Long Li, Riguang Liu and Jiangwei Li carried out the data collection, data analysis, and revised the paper. All authors reviewed the results and approved the final version of the manuscript.}

\section{Funding}

This work was supported by The Doctoral Research Project Fund of Guizhou Medical University (Grant No. Yuan Bo He J Zi [2015] 019); the Scientific Research Cooperation Project of the Science and Technology Agency of Guizhou Province (Grant No. Qian Ke He LH Zi [2016] 7228); the Science and Technology Cooperation Project of the Science and Technology Agency of Guizhou Province and Guizhou Medical University (Grant No. Qian Ke He Ping Tai Ren Cai [2018]5779-49); and the Scientific Research Project of the Science and Technology Agency of Guizhou Province (Grant No. Qian Ke He Ji Chu [2019]1265). The funders had no role in study design, data collection and analysis, decision to publish, or preparation of the manuscript.

\section{Availability of data and materials}

The datasets used and/or analyzed during the current study are available from the corresponding author on reasonable request.

\section{Ethics approval and consent to participate}

The trial was conducted in accordance with the Declaration of Helsinki. The study was approved by the Ethics Committee of the Affiliated Hospital of Guizhou Medical University (Approval No. 2018 Lunshen [15]) and registered for clinical trials in ChiCTR (http://www.chictr.org.cn): ChiCTR1800017066. And informed consent was taken from all the patients, the subjects or family members were briefed on the study aims and rationale, and this explanation provided them an understanding of the study prior to them signing the consent document.

\section{Consent for publication}

Not applicable.

\section{Competing interests}

The authors declare that they have no competing interests.

Received: 5 December 2020 Accepted: 3 February 2021

Published online: 11 February 2021

\section{References}

1. Musumeci G, Trovato FM, Loreto C, et al. Lubricin expression in human osteoarthritic knee meniscus and synovial fluid: a morphological, immunohistochemical and biochemical study. Acta Histochem. 2014 Jun; 116(5):965-72.

2. Safiri S, Kolahi A-A, Smith E, et al. Global, regional and national burden of osteoarthritis 1990-2017: a systematic analysis of the global burden of disease study 2017. Ann Rheum Dis. 2020;78(11):1463-71.

3. Musumeci G, Carnazza ML, Loreto C, et al. $\beta$-Defensin-4 (HBD-4) is expressed in chondrocytes derived from normal and osteoarthritic cartilage encapsulated in PEGDA scaffold. Acta Histochem. 2012 Dec;114(8):805-12.

4. Castrogiovanni P, Di Rosa M, Ravalli S, et al. Moderate physical activity as a prevention method for knee osteoarthritis and the role of synoviocytes as biological key. Int J Mol Sci. 2019;20(3):511. https://doi.org/10.3390/ijms2003 0511.

5. Rice D, McNair P, Huysmans E, Letzen J, Finan P. Best evidence rehabilitation for chronic pain part 5: osteoarthritis. J Clin Med. 2019;8(11):1769. https:// doi.org/10.3390/jcm8111769.

6. Oliveira MC, Vullings J, van de Loo FAJ. Osteoporosis and osteoarthritis are two sides of the same coin paid for obesity. Nutrition. 2020;70:110486

7. Deveza LA, Nelson AE, Loeser RF. Phenotypes of osteoarthritis: current state and future implications. Clin Exp Rheumatol. 2019;37(Suppl 120):64-72.

8. Ghouri A, Conaghan PG. Treating osteoarthritis pain: recent approaches using pharmacological therapies. Clin Exp Rheumatol. 2019;37(Suppl 120): 124-9.

9. Park NR, Lim KE, Han MS, Che X, Park CY, Kim JE, et al. Core binding factor beta plays a critical role during chondrocyte differentiation. J Cell Physiol. 2016:231:162-71.

10. Tahirov TH, Bushweller J. Structure and biophysics of CBFbeta/RUNX and its translocation products. Adv Exp Med Biol. 2017;962:21-31.

11. LeBlanc KT, Walcott ME, Gaur T, O'Connell SL, Basil K, Tadiri CP, et al. RunX activities in superficial zone chondrocytes, osteoarthritic chondrocyte clones and response to mechanical loading. J Cell Physiol. 2015;230:440-8.

12. Kundu M, Javed A, Jeon JP, Horner A, Shum L, Eckhaus M, et al. Cbfbeta interacts with Runx2 and has a critical role in bone development. Nat Genet. 2002;32:639-44.

13. Johnson K, Zhu S, Tremblay MS, Payette JN, Wang J, Bouchez LC, et al. A stem cell-based approach to cartilage repair. Science. 2012;336:717-21.

14. Kellgren JH, Lawrence JS. Radiological assessment of osteo-arthrosis. Ann Rheum Dis. 1957;16:494-502.

15. Musumeci G, Castrogiovanni P, Trovato FM, Weinberg AM, Al-Wasiyah MK, Alqahtani $\mathrm{MH}$, et al. Biomarkers of chondrocyte apoptosis and autophagy in osteoarthritis. Int J Mol Sci. 2015;16:20560-75.

16. Zamli Z, Sharif M. Chondrocyte apoptosis: a cause or consequence of osteoarthritis? Int J Rheum Dis. 2011;14:159-66.

17. Musumeci G, Loreto C, Carnazza ML, Strehin I, Elisseeff J. OA cartilage derived chondrocytes encapsulated in poly (ethylene glycol) diacrylate 
(PEGDA) for the evaluation of cartilage restoration and apoptosis in an in vitro model. Histol Histopathol. 2011;26:1265-78.

18. Thomas CM, Murray R, Sharif M. Chondrocyte apoptosis determined by caspase-3 expression varies with fibronectin distribution in equine articular cartilage. Int J Rheum Dis. 2011;14:290-7.

19. Musumeci G, Castrogiovanni P, Mazzone V, Szychlinska MA, Castorina S, Loreto C. Histochemistry as a unique approach for investigating normal and osteoarthritic cartilage. Eur J Histochem. 2014:58:2371.

20. Tian F, Wu M, Deng L, Zhu G, Ma J, Gao B, et al. Core binding factor beta (Cbfbeta) controls the balance of chondrocyte proliferation and differentiation by upregulating Indian hedgehog (Ihh) expression and inhibiting parathyroid hormone-related protein receptor (PPR) expression in postnatal cartilage and bone formation. J Bone Miner Res. 2014;29:1564-74.

21. Miller J, Horner A, Stacy T, Lowrey C, Lian JB, Stein G, et al. The core-binding factor beta subunit is required for bone formation and hematopoietic maturation. Nat Genet. 2002;32:645-9.

22. Henrotin Y, Sanchez C, Bay-Jensen AC, Mobasheri A. Osteoarthritis biomarkers derived from cartilage extracellular matrix: current status and future perspectives. Ann Phys Rehabil Med. 2016;59:145-8.

23. Sanchez C, Bay-Jensen AC, Pap T, Dvir-Ginzberg M, Quasnichka H, BarrettJolley $R$, et al. Chondrocyte secretome: a source of novel insights and exploratory biomarkers of osteoarthritis. Osteoarthr Cartil. 2017;25:1199-209.

24. Verma P, Dalal K. Serum cartilage oligomeric matrix protein (COMP) in knee osteoarthritis: a novel diagnostic and prognostic biomarker. J Orthop Res. 2013;31:999-1006.

25. Volck B, Ostergaard K, Johansen JS, Garbarsch C, Price PA. The distribution of YKL-40 in osteoarthritic and normal human articular cartilage. Scand J Rheumatol. 1999;28:171-9.

26. Martin-Millan M, Castaneda S. Estrogens, osteoarthritis and inflammation Joint Bone Spine. 2013;80:368-73.

27. Davies CM, Guilak F, Weinberg JB, Fermor B. Reactive nitrogen and oxygen species in interleukin-1-mediated DNA damage associated with osteoarthritis. Osteoarthr Cartil. 2008;16:624-30.

28. Ruan $\mathrm{G}, \mathrm{Xu}$ J, Wang $\mathrm{K}, \mathrm{Wu}$ J, Zhu Q, Ren J, et al. Associations between knee structural measures, circulating inflammatory factors and MMP13 in patients with knee osteoarthritis. Osteoarthr Cartil. 2018;26:1063-9.

29. Loreto C, Leonardi R, Musumeci $G$, et al. An ex vivo study on immunohistochemical localization of MMP-7 and MMP-9 in temporomandibular joint discs with internal derangement. Eur J Histochem. 2013 Apr 15;57(2):e12.

30. Leonardi R, Loreto C, Barbato E, et al. MMP-13 (collagenase 3) localization in human temporomandibular joint discs with internal derangement. Acta Histochem. 2008;110(4):314-8.

31. Haneda M, Hayashi S, Matsumoto T, Hashimoto S, Takayama K, Chinzei N, et al. Depletion of aquaporin 1 decreased ADAMTS4 expression in human chondrocytes. Mol Med Rep. 2018;17:4874-82.

32. Zhong L, Schivo S, Huang $X$, et al. Nitric oxide mediates crosstalk between interleukin $1 \beta$ and WNT signaling in primary human chondrocytes by reducing DKK1 and FRZB expression. Int J Mol Sci. 2017;18(11):2491. https:// doi.org/10.3390/ijms 18112491.

33. Ozler K, Aktas E, Atay C, Yilmaz B, Arikan M, Gungor S. Serum and knee synovial fluid matrixmetalloproteinase-13 and tumor necrosis factor-alpha levels in patients with late stage osteoarthritis. Acta Orthop Traumatol Turc. 2016:50:670-3.

34. Sasaki K, Yagi H, Bronson RT, Tominaga K, Matsunashi T, Deguchi K, et al. Absence of fetal liver hematopoiesis in mice deficient in transcriptional coactivator core binding factor beta. Proc Natl Acad Sci U S A. 1996;93: 12359-63.

35. Komori T. Roles of Runx2 in skeletal development. Adv Exp Med Biol. 2017; 962:83-93.

36. Yoshida CA, Furuichi T, Fujita T, Fukuyama R, Kanatani N, Kobayashi S, et al. Core-binding factor beta interacts with Runx2 and is required for skeletal development. Nat Genet. 2002;32:633-8.

37. Komori T. Runx2, an inducer of osteoblast and chondrocyte differentiation. Histochem Cell Biol. 2018;149:313-23.

\section{Publisher's Note}

Springer Nature remains neutral with regard to jurisdictional claims in published maps and institutional affiliations.

Ready to submit your research? Choose BMC and benefit from:

- fast, convenient online submission

- thorough peer review by experienced researchers in your field

- rapid publication on acceptance

- support for research data, including large and complex data types

- gold Open Access which fosters wider collaboration and increased citations

- maximum visibility for your research: over $100 \mathrm{M}$ website views per year

At BMC, research is always in progress.

Learn more biomedcentral.com/submissions 